\title{
Particle Identification for Physics beyond the LHC
}

\author{
Marco Battaglia \\ CERN, Geneva, Switzerland and University of California, Berkeley, USA
}

\begin{abstract}
Accelerator physics beyond the LHC is expected to provide precision in the study of new physics processes which the LHC may have already unveiled and to extend the high energy frontier beyond its reach, in the multi-TeV domain. In this paper I review the anticipated needs in terms of particle identification of this physics program in relation to the experimental conditions.
\end{abstract}

Key words:

PACS:

\section{Introduction}

Charged particle identification has been, and still is, crucial for detecting new signals and performing precision measurements at collider experiments. At LEP and SLC the identification of leptons has been essential. That of hadrons has proven to be beneficial to carry out the physics program in full.

The focus on heavy flavour physics and the study of $\mathrm{CP}$ violation in the $B$ sector further underlines the need for kaon and proton tagging. $B$ experiments are all equipped with Cherenkov detectors to tackle problems such as the discrimination of penguin-mediated $b \rightarrow s$ transitions from tree-level $b \rightarrow u$ processes. At the LHC proton collider, the hadron identification capabilities are similarly em-

Email address: marco.battaglia@cern.ch (Marco Battaglia). phasised by the specialised experiments. Lepton identification is an essential component in the design of multi-purpose experiments, from the Level1 trigger stage.

The LHC is expected to provide a major breakthrough in particle physics, by probing the Higgs sector and testing new physics beyond the Standard Model (SM), possibly manifesting the existence of Supersymmetry. But to understand the mechanism of origin of mass and electro-weak symmetry breaking, neither the study of the Higgs profile nor the search for additional Higgs particles will be completed at the LHC . Some measurements are limited in accuracy while others may not be feasible at all. In particular, understanding Supersymmetry requires to identify and measure all the supersymmetric particle partners. More generally, given a set of signals at LHC, their nature and properties may not be unambiguously determined, unless additional data is provided by a new gen- 
eration of particle colliders. This paper addresses some of the issues in particle identification which emerge from the anticipated physics programs of future colliders, with emphasis on $e^{+} e^{-}$linear colliders.

\section{Accelerator Designs and Opportunities}

There are several alternative paths for reaching the high energy frontier, beyond the LHC . The luminosity in proton collisions can be increased. The LHC can be upgraded to provide collisions at $10^{35} \mathrm{~cm}^{-2} \mathrm{~s}^{-1}$. Such upgrade (SLHC) could extend the mass reach to new particles by about $30 \%$, provided the ATLAS and CMS detectors can be refitted to cope with the higher particle fluxes. But past $10^{35} \mathrm{~cm}^{-2} \mathrm{~s}^{-1}$, the LHC detector performance greatly degrades, even with major upgrades [1]. At these luminosities, particle identification techniques such as transition radiation detectors appear to be not applicable. An increase in $\sqrt{s}$ should be significantly easier to be exploited by the experiments. This makes a very large hadron collider (VLHC ) a possible long-term option for accelerator HEP [2]. A large $\sqrt{s}$ increase from the LHC energy has to be supported by very significant $R \& D$ for magnets and vacuum. An $e^{+} e^{-}$linear collider (LC), providing collisions at energies from the $Z^{0}$ pole up to approximately $1 \mathrm{TeV}$ with luminosity in excess to $10^{34} \mathrm{~cm}^{-2} \mathrm{~s}^{-1}$, is presently considered as the best motivated option for the next large scale project in accelerator particle physics. The linear collider R\&D is reaching the time at which a decision on the technical feasibility of the project and an informed choice of the most advantageous technology can be taken [3]. Such collider would not directly test the energy range beyond the LHC , but it will complement its data with the accuracy characteristic of lepton colliders. Through the collision of point-like particles with tunable energies, the democratic production of particles, including those interacting only weakly, and the possibility to modify their polarisation, as well as to replace $e^{+} e^{-}$with $\gamma \gamma$ and $e^{-} e^{-}$collisions, the high energy linear collider offers a unique opportunity. Lepton collisions are not limited to the $\mathrm{TeV}$ fron-
Table 1

Track density and tracker occupancy (normalised to LHC ) in proton collisions from the LHC to the VLHC

\begin{tabular}{|l|cccc|}
\hline & \multicolumn{4}{|c|}{ Lhc SLHC VLHC -I VLHC -II } \\
\hline$\sqrt{s}(\mathrm{TeV})$ & 14 & 14 & 40 & 200 \\
$d N / d \eta / \mathrm{BX}$ & 150 & 750 & 180 & 500 \\
$\left\langle E_{T}\right\rangle(\mathrm{GeV})$ & 0.5 & 0.5 & 0.5 & 0.6 \\
Tracker Occupancy & 1 & 10 & 1 & 3 \\
\hline
\end{tabular}

tier. Active $\mathrm{R} \& \mathrm{D}$ programs are already addressing the challenges offered by multi- $\mathrm{TeV}$ collisions with electrons (CLIC) and, in a longer perspective, possibly also with muons, and providing conceptual designs. Hadron and Lepton Colliders give an healthy complementarity of approaches and technological challenges which are being addressed by a significant, continued world-wide R\&D effort.

\section{Physics Program and Particle Identification}

The details of the physics program for the next generation of particle colliders will be defined by the exploration of the $\mathrm{TeV}$ frontier by the LHC . Our present understanding indicates that the study of the Higgs sector will require efficient flavour tagging, to determine its quark couplings, efficient lepton identification for mass, spin and gauge couplings measurements. Several models of new physics which address the hierarchy problem are characterised by a rich spectroscopy of new states, with abundance of multi-lepton signatures.

The SLHC will requires major tracker rebuilds to maintain jet tagging capabilities and fully benefit from the higher luminosity. Moving to higher energies poses problems which become particularly severe in the forward regions where jet and lepton tagging are essential.

In this environment lepton identification needs to be guaranteed as multi-lepton signatures will represent an important handle in isolating signals of new physics. A good example is offered by $W W$ and $Z Z$ vector boson scattering. If no elementary Higgs boson exists, electroweak symmetry breaking should happen dynamically. The effects from 
strong boson scattering should become observable at the SLHC with good statistical significance analysing the leptonic decays of the gauge bosons. These studies will have to rely on efficient and well modelled response functions for lepton identification in the high luminosity environment.

The $e^{+} e^{-}$linear collider offers more benign experimental conditions to consider advanced particle identification techniques with a wide array of physics opportunities and aims at high precision measurements. It thus offer important options for characterising the particle identification needs of collider physics beyond the LHC. Electron identification can be obtained from the response of the finely segmented electromagnetic calorimeter. Muons are identified in the instrumented return flux iron. Plastic streamer tubes, or RPC detectors, can be used to provide multiple samplings since rates are low. The typical momentum of leptons in muons in jets is $\simeq 20 \mathrm{GeV}$, corresponding to a lateral spread of $\simeq 2 \mathrm{~cm}$ after $7 \lambda$, which sets the scale of the needed position resolution. Assuming a solenoidal magnetic field of 4 Tesla, the muon momentum cut-off is $\simeq 5 \mathrm{GeV}$ for a coil located at $4 \mathrm{~m}$ from the interaction region.

Charged particle identification offers crucial sensitivity to new phenomena and provides redundancy in discriminating signals for precision measurements. In principle, it would be advantageous to provide the LC experiment with both lepton and hadron tagging capabilities. However, analysing the multi-jet final states requires excellent parton energy and direction reconstruction. This can be best achieved with the energy flow technique, which combines tracking and calorimetric information. There is a need to minimise the material in front of the electro-magnetic calorimeter which imposes some compromises. In absence of a clear case for hadron identification with good purity, current detector designs are relying on the information which can be obtained from specific ionization, $\mathrm{dE} / \mathrm{dx}$, in the Main Tracker device for hadron identification. Both a large volume Time Projection Chamber (TPC) and a Si tracker are being considered. The TPC design included in the TESLA proposal has 240 points/track with micro-pattern gaseous detector read-out. A dE/dx resolution of $\simeq 4 \%$ is achievable, corresponding

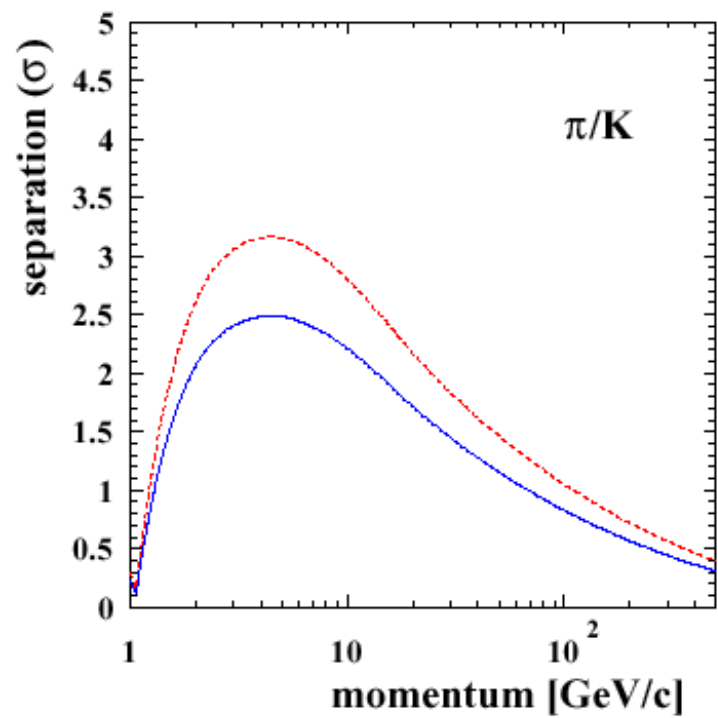

Fig. 1. Hadron identification with $d E / d x$ in TPC at the Linear Collider: $\pi / K$ separation as function of particle momentum in units of $\sigma$ for the proposed TESLA detector (from [4]).

to $\mathrm{a} \geq 2.0 \sigma \pi / K$ separation in the kinematic region of $1.5 \mathrm{GeV}<p<20 \mathrm{GeV}$ (see Figure 1) [4]. Together with the excellent mass resolution afforded by the accurate momentum determination, this would ensure a good separation of processes such as $B \rightarrow \pi \pi$ at a Giga- $Z$ factory. A discrete $\mathrm{Si}$ Tracker may provide $\mathrm{dE} / \mathrm{dx}$ measurements in $\mathrm{Si}$, as pioneered by DELPHI and Belle. However, the resolution is much poorer, due to limited number of samplings available, and hadron tagging would only be possible in the $1 / \beta^{2}$ region, since the relativistic rise saturates.

Further specifications for hadron identification may come from physics at the $W^{+} W^{-}$threshold where some of the elements of the CKM mixing matrix could be directly determined, with interesting accuracy if $c$ and $s$ jets can be efficiently tagged. Such direct measurements will be free from the theoretical uncertainties and model dependence which affect the determination derived from partial decay widths. This would call for good $K$ identification over a wide momentum range. However, at higher energies benefits from $c$ and $s$ tagging in $W^{ \pm}$boson decays, appear to be marginal. The study of $W_{L} W_{L}$ production represents a good test case. An 
increase of longitudinally polarised $W$ pair production in high energy $e^{+} e^{-}$collisions is predicted some models, including technicolor. The helicity of $W$ bosons can be best measured in mixed $e^{+} e^{-} \rightarrow$ $W W \rightarrow \ell \nu c \bar{s}$ decays where the lepton charge and the flavour of the $c$ quark can be used as analysers. Charged kaon identification enhances the tagging quality. But including realistic performances dilutes such improvement to only $\simeq 20 \%$ [5]. However, we should remain aware that the most exciting part of the physics program at future colliders may come from physics which we cannot anticipate today. Therefore the detector concepts should ensure that some redundancy in the tagging capabilities is kept.

The case for excellent lepton identification is easily made, since it is instrumental to some of the most fundamental processes on the linear collider agenda. The study of the Higgs boson through the signature process $e^{+} e^{-} \rightarrow Z^{0} H^{0}$ relies on tags of the leptonic $Z^{0}$ decay to perform studies independent on the Higgs decay properties. Since these leptons are quite energetic, their identification is ensured through the electro-magnetic, hadronic calorimeter and muon chamber response. But other cases are less straightforward. A detailed study of Higgs boson couplings to fermions and gauge bosons, requires the use of inclusive four jet events. Here semi-leptonic $b$-quark decays giving will distort the $M_{J J}$ invariant mass distribution. It is thus important to tag soft leptons in jets to apply appropriate corrections. If new physics exists, to cancel the effect of heavy Higgs mass in electroweak observables, its mass $M_{H}$ can be significantly heavier than the $\simeq 210 \mathrm{GeV}$ limit derived from present data. In this case it would become interesting to search for $e^{+} e^{-} \rightarrow H^{0} e^{+} e^{-} \rightarrow$ $X e^{+} e^{-}$at high energies, which has a large cross section and allows model-independent analyses to be performed by tagging the forward electrons. The $Z Z$ fusion analysis needs to identify electrons and precisely measure their energy and direction, down to $\simeq 100 \mathrm{mrad}$, close to the bulk of the $\gamma \gamma$ background.

If Supersymmetry is realised in nature, the $e^{+} e^{-}$ collider is also expected to complement the LHC to determine its fundamental parameters and establish whether the lightest supersymmetric particle
(LSP) is indeed responsible for dark matter (DM) in the Universe. In particular, the LC will produce and measure accurately the $\tilde{\ell}$ sleptons and gauginos, including the LSP, properties. The recent WMAP satellite determination of DM density constrains the phase space of supersymmetric model parameters. In a constrained MSSM these data indicates that the supersymmetric spectrum is likely to have small slepton-LSP mass differences, corresponding to rather soft spectra of the lepton produced in $\tilde{\ell}$ decays $[6,7]$.

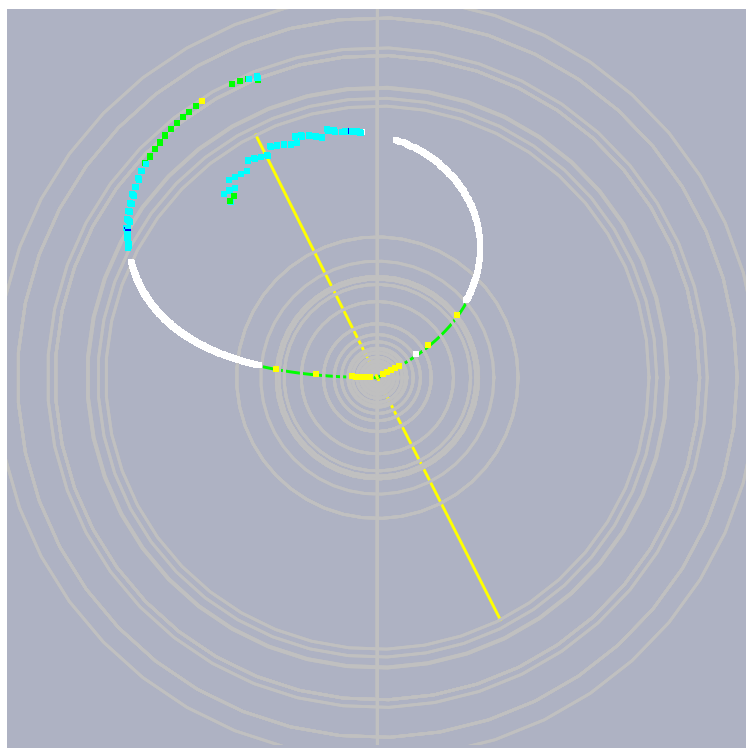

Fig. 2. Supersymmetric particle production with low momentum leptons: a fish-eye view of a $e^{+} e^{-} \rightarrow \tilde{\mu_{R}}{ }^{+} \tilde{\mu R}^{-} \rightarrow \mu^{+} \chi_{1}^{0} \mu^{-} \chi_{1}^{0}$ event at $\sqrt{s}=1 \mathrm{TeV}$. One muon is produced at the lowest momentum available to the reaction and is not reaching the muon detectors.

The slepton and neutralino masses can be accurately determined from the endpoints of the lepton momentum distribution in the two-body decay. But at the upper tip of the cosmologically interesting region, where the two particles are almost degenerate, the lower lepton energy edge may be located at about $1 \mathrm{GeV}$ (see Figures 2 and 3). This raises the issue of lepton tagging capabilities extending to such low energies matched with efficient event selection in presence of accelerator-induced backgrounds.

A similar scenario arises in a particular class of models with extra dimensions. Extra dimensions 


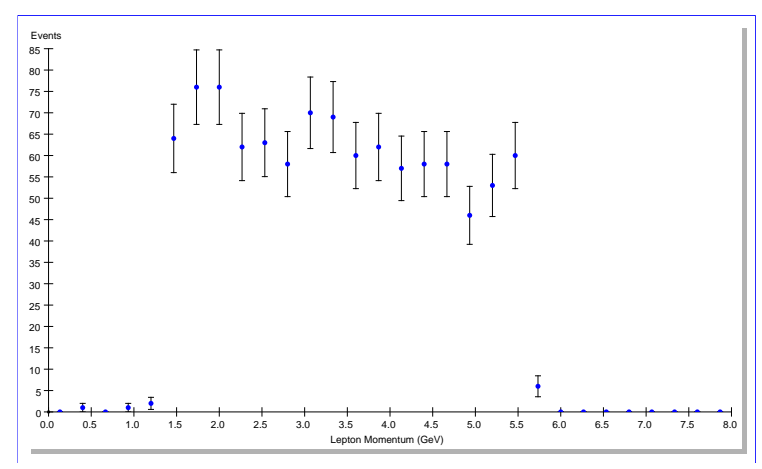

Fig. 3. Supersymmetric particle production with low momentum leptons: momentum distribution of leptons from $e^{+} e^{-} \rightarrow \tilde{\mu_{R}}{ }^{+} \tilde{\mu R}^{-} \rightarrow \mu^{+} \chi_{1}^{0} \mu^{-} \chi_{1}^{0}$ events at $\sqrt{s}=1 \mathrm{TeV}$. The $\tilde{\ell}$ and $\chi_{1}^{0}$ masses can be extracted from the location of the lower and upper kinematic endpoints.

are being actively explored as an alternative solution to the hierarchy problem. Most realisations are expected to give spectacular signals at future colliders. Universal extra dimension (UED) models have all SM particles propagating in one or more compact extra dimensions. Kaluza Klein partners of SM particles have masses $\simeq n R^{-1}$, where $R$ is the compactification radius, and identical spin and couplings to SM particles. The mass degeneracy of particles of the same KK-level $n$ is only broken by radiative corrections and KK-parity conservation guarantees that the lightest KK state, generally the photon or neutrino excitation, is stable. So UED also offers a viable CDM candidate in the form of its lightest KK particle [8]. This scenario can be tested in details at a LC of sufficient energy through processes where lepton KK excitations are pair produced to decay into ordinary leptons and stable lightest KK states. This closely resembles the slepton production and decay process in Supersymmetry, except that here the mass splitting is always small and measuring the lower lepton energy edge requires tagging soft muons and electrons.

Studying $e^{+} e^{-} \rightarrow f \bar{f}$ at $\sqrt{s} \geq 1$ Tev will provide a window on New Physics at scales of order $10 \mathrm{TeV}$ and more, which are much beyond those directly reachable at the next generations of colliders. But to ensure sensitivity to new phenomena through the electro-weak fits, it will be important to perform accurate forward-backward asymmetry measurements for both leptons and quarks, which ensure the best sensitivity at the upper scale edge [9]. The reaction $e^{+} e^{-} \rightarrow t \bar{t}$ at multi-TeV energies offers an interesting case. Here the charge of the quark can be determined from that of the lepton produced in the leptonic $W$ decay. Therefore, indirect sensitivity to the $10 \mathrm{TeV}$ scale will rely on efficient tagging of relatively soft leptons.
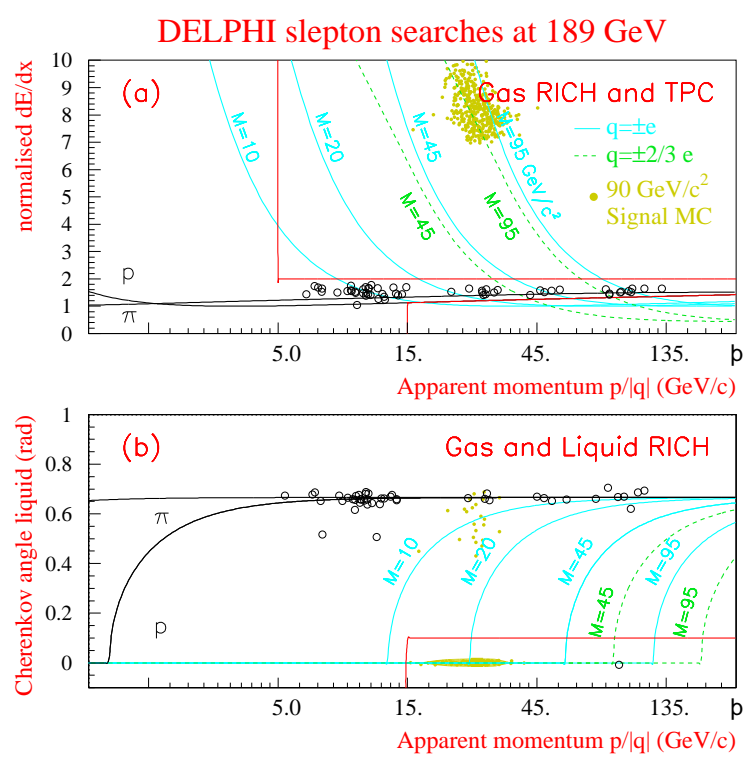

Fig. 4. Heavy stable particle search with DeLphi at LeP (from [10]).

New particles may also appear in the form of heavy stable particles, directly detectable with particle identification and time of flight (TOF). At a radius of $2 \mathrm{~m}$ the time-of-flight of a $\beta=1$ particle is $6.7 \mathrm{~ns}$ which can be measured with $\simeq 50 \mathrm{ps}$ accuracy. Since data is integrated over several bunch crossings in a train and, depending on RF chosen technique, the bunch spacing $\Delta t_{b}$ is in the range 1-300 ns, TOF must deal with ambiguities. The TPC may be used to resolve the bunch crossing by extrapolating to the collision point. An example is offered in the context of Supersymmetry by Gauge-mediated Supersymmetry Breaking Models (GMSB), where the gravitino, $\tilde{G}$ is the LSP. Due to its weak coupling, the next lightest particle (NLSP) is long-lived. The $\tilde{\tau}$ NLSP scenario with $\tilde{\tau} \rightarrow \tilde{G} \tau$ can be investigated in $\tilde{\tau}$ pair production. The signature is a pair of heavy long-lived charged particles traversing the detector. Their existence 
can be revealed through TOF and specific ionization, if not dedicated particle identification detectors, using a technique pioneered at LEP (see Figure 4) [10]. Care needs to be taken to ensure that the resulting large ionization signals are preserved by the dynamic range of the read-out electronics.

\section{Conclusion}

The future of particle physics depends on the availability of new generations of colliders after the LHC . The $e^{+} e^{-}$linear collider is the next large scale project under consideration. While hadron identification does not appear to have a major impact on the LC physics capabilities, identification of leptons, and also of new heavy stable hadrons, will be instrumental to many of its key physics processes. Examples from the main lines of investigations expected at present, indicate the challenging requirements from the wide range of kinematics offered by anticipated physics signatures.

\section{References}

[1] F. Gianotti et al., arXiv:hep-ph/0204087

[2] G. Ambrosio et al. [VLHC Design Study Group Collaboration], SLAC-R-591

[3] G. Loew, Nucl. Phys. Proc. Suppl. 117 (2003) 385.

[4] T. Behnke, S. Bertolucci, R.D. Heuer and R. Settles, "TESLA: The superconducting electron positron linear collider with an integrated X-ray laser laboratory. Technical design report. Part. 4: A detector for TESLA," DESY-01-011

[5] A. Soffer, in Proc. of the APS/DPF/DPB Summer Study on the Future of Particle Physics (Snowmass 2001) ed. N. Graf, eConf C010630, E3039 (2001).

[6] J.R. Ellis, K.A. Olive, Y. Santoso and V.C. Spanos, Phys. Lett. B 565 (2003) 176 arXiv:hep-ph/0303043.

[7] M. Battaglia, A. De Roeck, J.R. Ellis, F. Gianotti, K.A. Olive and L. Pape, arXiv:hep-ph/0306219

[8] G. Servant and T.M.P. Tait, Nucl. Phys. B 650 (2003) 391 arXiv:hep-ph/0206071.

[9] M. Battaglia, S. De Curtis and D. Dominici, JHEP 0212 (2002) 004 arXiv:hep-ph/0210351.
[10] P. Abreu et al. [DELPHI Collaboration], Phys. Lett. B 478 (2000) 65 arXiv:hep-ex/0103038. 